\title{
Temporal dynamics of animacy categorization in the brain of patients with mild cognitive impairment
}

Hamed Karimi ${ }^{1,2, *}$, Haniye Marefat ${ }^{3}$, Mahdiyeh Khanbagi' ${ }^{2}$, Chris Kalafatis ${ }^{4,5,6}$, Hadi Modarres $^{6}$, Zahra Vahabi ${ }^{7,8}$, Seyed-Mahdi Khaligh-Razavi²,6,"

${ }^{1}$ Department of Mathematics and Computer Science, Amirkabir University of Technology, Tehran, Iran

${ }^{2}$ Royan Institute for Stem Cell Biology and Technology, ACECR, Tehran, Iran

${ }^{3}$ School of Cognitive Sciences, Institute for Research in Fundamental Sciences (IPM), Tehran, Iran

${ }^{4}$ South London \& Maudsley NHS Foundation Trust, London, UK

${ }^{5}$ Department of Old Age Psychiatry, King's College London, London, UK

${ }^{6}$ Cognetivity Itd, London, UK

${ }^{7}$ Department of Geriatric Medicine, Ziaeian Hospital, Tehran University of Medical Sciences, Tehran, Iran

${ }^{8}$ Memory and Behavioral Neurology Division, Roozbeh Hospital, Tehran University of Medical Sciences, Tehran, Iran

\author{
${ }^{*}$ Corresponding authors: \\ Hamed Karimi \\ (hamedk72@gmail.com) \\ Seyed-Mahdi Khaligh-Razavi \\ (seyed@cognetivity.com)
}

Keywords: Electroencephalography, Mild Cognitive Impairment, Multivariate Pattern Analysis, Multivariate Decoding, Animacy Information Processing 


\section{Abstract}

Electroencephalography (EEG) has been commonly used to measure brain alterations in Alzheimer's Disease (AD). However, reported changes are limited to those obtained from using univariate measures, including activation level and frequency bands. To look beyond the activation level, we used multivariate pattern analysis (MVPA) to extract patterns of information from EEG responses to images in an animacy categorization task. Comparing healthy controls $(\mathrm{HC})$ with patients with mild cognitive impairment $(\mathrm{MCl})$, we found that the neural speed of animacy information processing is decreased in $\mathrm{MCl}$ patients. Moreover, we found critical time-points during which the representational pattern of animacy for $\mathrm{MCl}$ patients was significantly discriminable from that of $\mathrm{HC}$, while the activation level remained unchanged. Together, these results suggest that the speed and pattern of animacy information processing provide clinically useful information as a potential biomarker for detecting early changes in $\mathrm{MCl}$ and $\mathrm{AD}$ patients. 


\section{Introduction}

Mild Cognitive Impairment $(\mathrm{MCl})$ is a condition in which an individual has a mild but measurable decline in cognitive abilities. This decline is noticeable to the person affected and to the family members and friends, but the individual can still carry out everyday activities $(1,2)$. A systematic review of 32 cohort studies shows an average of 32 percent conversion from $\mathrm{MCl}$ to Alzheimer's Disease (AD) within a five-year follow-up (3). 5-15 $\%$ of people with $\mathrm{MCl}$ have also shown to develop dementia every year (4).

Electroencephalography (EEG) is widely used to study the resting-state neural activity in the brain of patients with $\mathrm{MCl}$ and mild $\mathrm{AD}$ (5-11). A few studies have also used EEG to associate abnormalities in memory function with cognitive impairment during both encoding and decoding stages of working memory $(7,12-14)$ as well as episodic memory tasks (15).

The relationship between cognitive impairment and visual system changes has recently gained attention (16). Several studies have linked deficiencies in different parts of the visual system with $A D$ (17-19). There are several documented cases in which visual function problems are the initial and dominant manifestation of dementia $(20,21)$. A few studies have also used a visual task to report changes in the EEG responses of patients with $\mathrm{MCl}$ and $\mathrm{AD}(22,23)$.

Several studies of the visual system in primates and healthy human subjects have demonstrated that images are categorized by their animacy status (i.e. animate vs. inanimate) in the higher-level visual areas (24-29). The neural activity underlying the animacy information processing of briefly flashed images was also studied in healthy adults $(30,31)$. Studies have shown that animacy information emerges in the brain of healthy human subjects as early as $80 \pm 20 \mathrm{~ms}$ after the stimulus onset and reaches its peak within $250 \pm 50 \mathrm{~ms}$ after the stimulus onset (32-34). However, the underlying neural dynamics of animacy processing in the brain of patients with $\mathrm{MCl}$ in comparison to healthy controls $(\mathrm{HC})$ is still unknown.

The Integrated Cognitive Assessment (ICA) is a visual task based on a rapid categorization of natural images of animals and non-animals (35). ICA assesses changes in the speed and accuracy of animacy processing in patients with $\mathrm{MCl}$ and mild $\mathrm{AD}$, and is shown to be sensitive in detecting $\mathrm{MCl}$ patients $(36,37)$.

Here we acquired EEG data from $\mathrm{MCl}$ and $\mathrm{HC}$ participants during the completion of the ICA's animal/non-animal categorization task. We studied the temporal neural dynamics of animacy processing in $\mathrm{MCl}$ patients using both univariate and multivariate analyses. By applying multivariate pattern analysis (MVPA), we compared the neural speed of animacy processing in $\mathrm{MCl}$ and healthy individuals. We further looked beyond the 
conventional univariate methods and compared $\mathrm{MCl}$ and $\mathrm{HC}$ in terms of their pattern of EEG responses to natural images of animal and non-animal stimuli.

We find that the categorical representation of animacy information emerges later in the brain of patients with $\mathrm{MCl}$ compared to that of $\mathrm{HC}$. Furthermore, the results reveal differences between the EEG response patterns of $\mathrm{HC}$ vs. $\mathrm{MCl}$ during the time-points when univariate mean responses showed no significant difference. The EEG response patterns could further be used to discriminate $\mathrm{HC}$ from $\mathrm{MCl}$, demonstrating that the pattern of EEG activity also carries information about the status of the disease, beyond the conventional univariate analysis of mean activities.

\section{Methods}

\subsection{Integrated Cognitive Assessment (ICA) Task}

ICA $(35,38,39)$ is a rapid animal vs. non-animal categorization task. The participants are presented with natural images of animals and non-animals. The images are followed by a short blank screen and then a dynamic mask. Participants should categorize the images as animal or non-animal as quickly and accurately as possible (Figure 1). See (KhalighRazavi et al. 2019, Figure 1B) for sample images of the task.

\subsection{Montreal cognitive assessment (MoCA)}

MoCA (40) is a ten-minute pen and paper test with a maximum score of 30 and is conventionally used to assess visuospatial, memory, attention, and language abilities to detect cognitive impairment in older adults. An examiner is needed to administer the test.

\subsection{Addenbrooke Cognitive Examination (ACE-R)}

ACE-R (41) is another pen-and-paper tool for cognitive assessment with a maximum score of 100. It mainly assesses five cognitive domains: attention, orientation, memory, fluency, language, and visuospatial. On average, the test takes about 20 to 30 minutes to administer and score. 


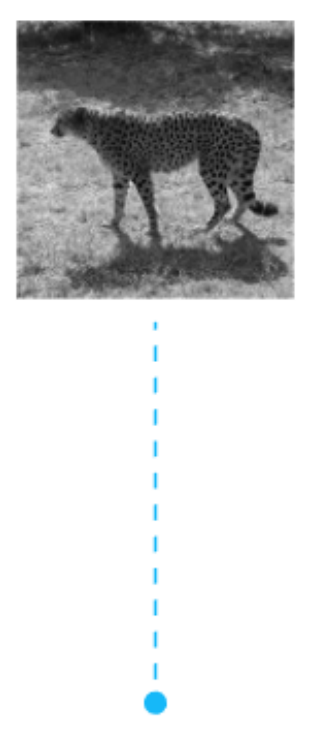

Stimulus

(100 ms)

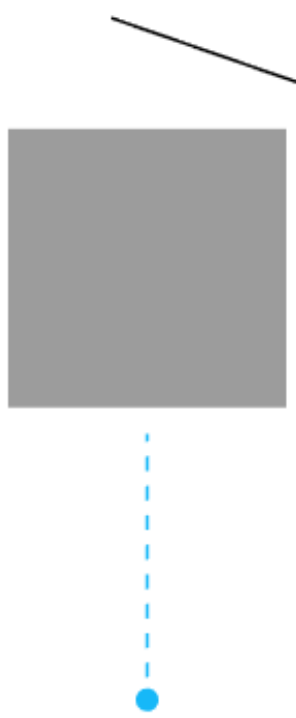

ISI

(20 ms)

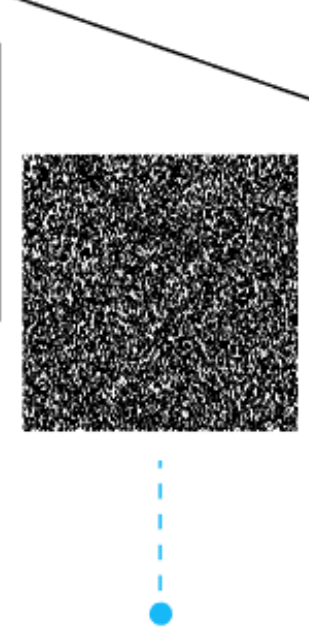

Dyanamic Mask (250 ms)
Figure 1. The EEG task. The EEG experiment contained 13 experimental runs; in each run, 32 natural images (16 animal, 16 non-animal) were presented to the participant in random order. Each image was shown for $100 \mathrm{~ms}$, followed by an interstimulus interval (ISI) of $20 \mathrm{~ms}$ and a dynamic noise mask for 250ms. Participants were given $1500 \mathrm{~ms}$ to indicate (using pre-specified buttons) whether the presented image contained an animal or not.

\subsection{Subject Recruitment}

40 (22 Healthy, $18 \mathrm{MCl}$ ) participants (Table 1) completed the ICA test, MoCA, and ACE$R$ in the first assessment. The participants were non-English speakers, with instructions for the cognitive assessments provided in Farsi. The ICA test was administered on an iPad.

Patients were recruited into the study prospectively. A consultant neurologist made all the diagnoses according to diagnostic criteria described by the working group formed by the National Institute of Neurological and Communicative Disorders and Stroke (NINCDS) and the Alzheimer's Disease and Related Disorders Association (ADRDA) (referred to as the NINCDS-ADRDA criteria) and the National Institute on Aging and Alzheimer's Association (NIA-AA) diagnostic guidelines. The study was conducted at the Royan Institute, according to the Declaration of Helsinki and approved by the local ethics committee at the Institute. The inclusion/exclusion criteria are listed below.

- Inclusion criteria for the control group:

Males and females aged between $50-85$ years who are not currently on medication that may interfere with the study results and are in good general health were included in the study. 
Table 1. Demographic information of participants

$S D$ : standard deviation

$p$-values were calculated from two-sided t-test for two independent samples

\begin{tabular}{|c|c|c|c|}
\hline Characteristic & $\mathrm{HC}(n=22)$ & $\mathrm{MCl}(n=18)$ & $P$-values \\
\hline Age -mean years \pm SD & $63.23 \pm 6.18$ & $63.55 \pm 6.40$ & 0.87 \\
\hline Education in years -mean \pm SD & $15 \pm 4.18$ & $14.72 \pm 5.02$ & 0.85 \\
\hline Gender (\%female) & $13(59 \%)$ & $10(55 \%)$ & 0.82 \\
\hline
\end{tabular}

- Inclusion criteria for the $\mathrm{MCl}$ group:

Males and females aged between $50-85$ years with a clinical diagnosis of $\mathrm{MCl}$ who were able to provide informed consent were included in the study.

- Exclusion criteria for both groups:

Individuals with a presence of significant cerebrovascular disease or major psychiatric disorder (e.g., Chronic psychosis, recurrent depressive disorder, generalized anxiety disorder, and bipolar mood disorder) or major medical comorbidities (e.g., Congestive Cardiac Failure, Diabetes Mellitus with renal impairment) were excluded from the study.

Additional exclusion criteria were: use of cognitive-enhancing drugs (e.g., cholinesterase inhibitors), or a concurrent diagnosis of epilepsy or any history of alcohol misuse, illicit drug abuse, severe visual impairment (e.g., macular degeneration, diabetic retinopathy, as determined by the clinical examination), or repeated head trauma.

\subsection{EEG Data Acquisition and Preprocessing}

The EEG experiment included a short version of the ICA task (i.e., smaller image set). Participants completed one EEG session that included 13 runs; each run lasted 67 seconds, during which 32 images were presented in random order, and participants had a short break in between the runs. Each stimulus is repeated 13 times during the whole EEG session (once in each run). These are referred to as repetition trials throughout the 
manuscript. Participants 16 and 17 completed 10 runs, and participants 12 and 22 completed 12 runs. We acquired the EEG data using a 64-channel (63 electrodes on the cap and one as the reference) g.tec product at a sampling rate of $1200 \mathrm{~Hz}$. Three electrooculograms (EOG) channels were set up to capture eye blinks.

The preprocessing consisted of six general steps, which were all done using Brainstorm (42) in MATLAB:

1. Re-referencing the data with the mean activation and removing the reference channel (channel 33).

2. Neutralizing eye blinks by removing the most correlated component with the EOG channels, using the independent component analysis algorithm.

3. Extracting pre-stimulus data from $100 \mathrm{~ms}$ before to $800 \mathrm{~ms}$ after the stimulus onset (epoching).

4. Normalizing the epochs regarding the mean and standard deviation of the baseline.

5. Smoothing the data with a $50 \mathrm{~Hz}$ low-pass filter.

6. Resampling the data to $1000 \mathrm{~Hz}$ sampling rate.

\subsection{Univariate Pattern Analysis - Event-Related Potential (ERP)}

We calculated the ERPs of the extracted epochs (from 100ms before to $800 \mathrm{~ms}$ after the stimulus onset) by averaging the EEG responses to all stimuli within each group of channels. We calculated the ERPs separately for each individual. The ERP of HC and $\mathrm{MCl}$ are the average ERP of corresponding participants.

\subsection{Multivariate Pattern Analysis - Animal vs. non-Animal Decoding}

To study the emergence of animacy categorical information in the brain, we used a linear classifier to discriminate EEG responses to animal stimuli from that of non-animal (Figure 2). Before the classification, we randomly assigned each target stimulus with all its EEG trials to bins of size 2, 3, and 4 stimuli and randomly sub-averaged the trials within each bin. The decoding accuracy at each time-point ' $t$ ' is then the average accuracy of 10,000 repetitions in a leave-one-bin-out cross-validation model, using a support vector machine (SVM) classifier. The classification is done using the LIBSVM software implementation (43).

\subsection{Multivariate Pattern Analysis - Pairwise Decoding}

At each time-point, we measured the accuracy of an SVM classifier in discriminating pairs of stimuli using leave-one-out cross-validation. This leads to a symmetric $32 \times 32$ representational dissimilarity matrix $(\mathrm{RDM})$ at each time-point, representing the pairwise 


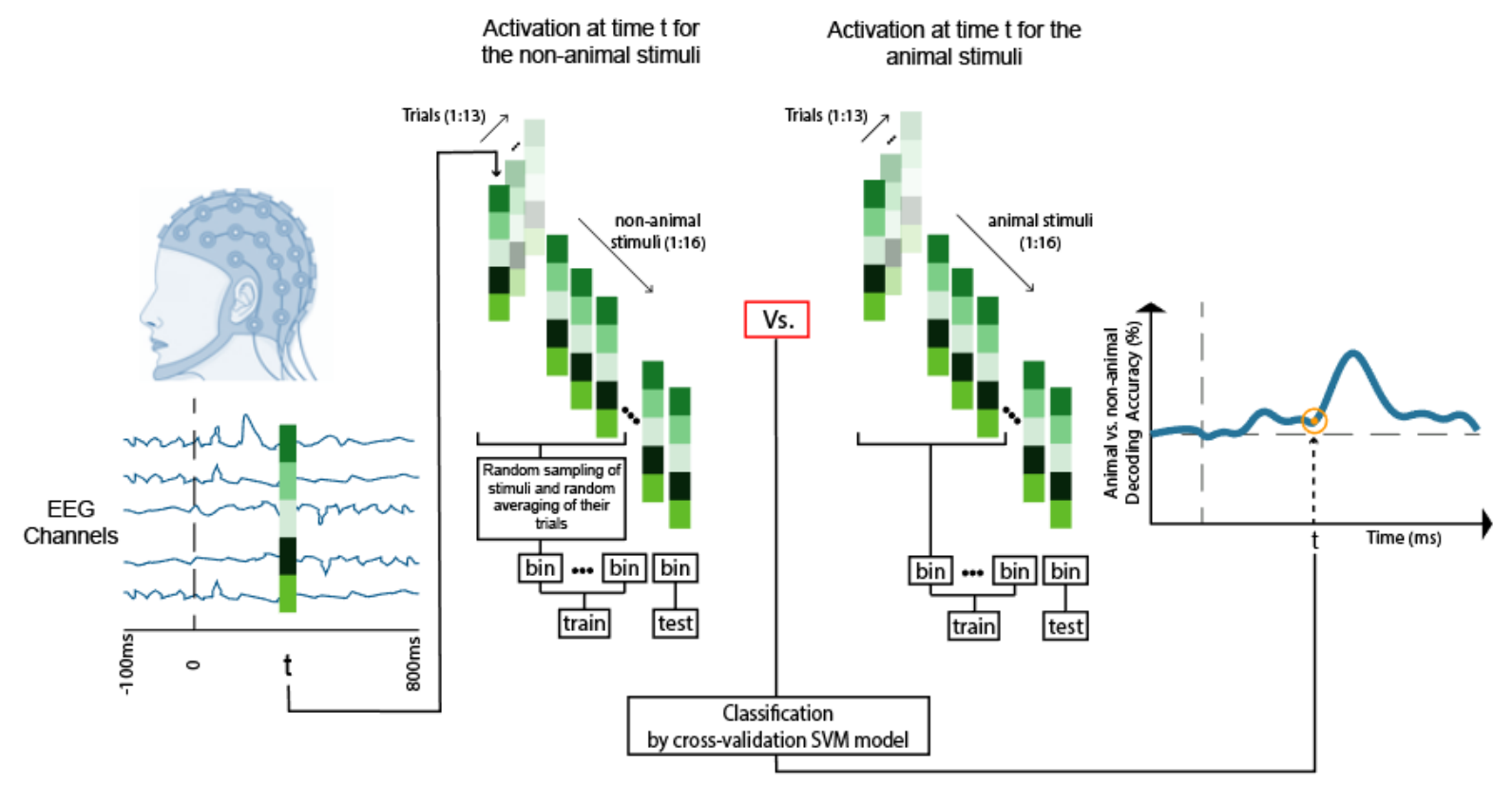

Figure 2. Animal vs. non-animal decoding. For each individual, we extracted the trials of EEG responses to animal and non-animal stimuli at each time-point (for a given time-point $t$ we have two matrices of 63 channels $x 16$ stimuli $x 13$ repetition trials for animal and non-animal stimuli, respectively). Before the classification, we randomly assigned each target stimulus with all its EEG trials to bins of size 2, 3 and 4 stimuli and randomly sub-averaged the trials within each bin. We trained a leave-one-bin-out cross-validation SVM model to discriminate animal from non-animal. At each timepoint, the decoding accuracy is the average of 10,000 repetitions of the classification procedure described above.

dissimilarities of stimuli in the off-diagonal elements (Figure 3). We repeated the entire process for each individual to create an RDM at each time-point.

\subsection{Multivariate Pattern Analysis - HC vs. MCl Classification}

We characterized the activation pattern at each time-point as a $63 \times 32$ matrix, with each column being the responses of the EEG channels to a stimulus, averaged over all repetition trials (Figure 4). We applied 10,000 bootstrap resampling (without replacement) of participants and trained a leave-one-out cross-validation SVM model to discriminate the EEG activation pattern of $\mathrm{HC}$ from that of $\mathrm{MCl}$.

\subsection{Multidimensional Scaling (MDS)}

Multidimensional Scaling (MDS) is a non-linear dimension reduction algorithm. It rearranges the data points in a p-dimensional space until their pairwise distance is consistent with a given dissimilarity matrix. Here, we used MDS to visualize the stimuli on a 2D plane based on their pairwise dissimilarity in RDMs. 


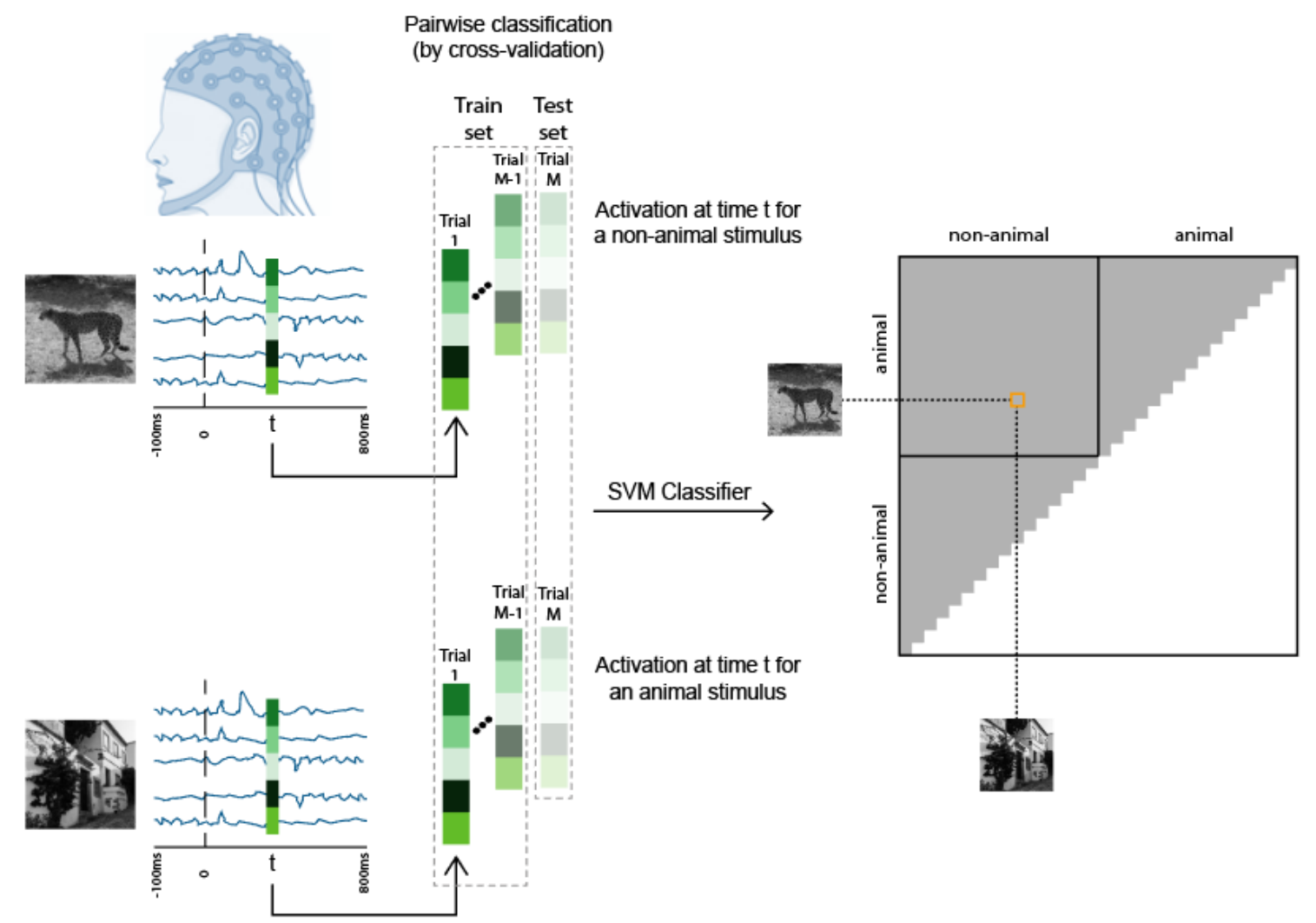

Figure 3. Representational dissimilarity matrices (RDMs). We trained an SVM to discriminate pairs of stimuli using their EEG responses at time-point $t$. This pairwise classification of stimuli by cross-validation SVM model leads to a $32 \times 32$ RDM at each time-point $t$.

\subsection{Statistical Analysis}

To avoid any assumption about the observed distributions, we only used non-parametric statistical tests. They are capable of testing a null hypothesis without any prior assumptions about the nature of the distribution:

\subsubsection{Bootstrap Test}

Bootstrapping is a strategy to estimate different statistics over an unknown distribution. It consists of a resampling (with or without replacement) procedure and applying a target function. The result is a bootstrap distribution that can be used for statistical inference purposes. We used 10,000 bootstrap resampling of participants without replacement from each group $(\mathrm{HC}$ and $\mathrm{MCl}$ ) and computed a $\mathrm{p}$-value as follows:

$$
p-\text { value }=\frac{\text { number of elements lower }(\text { or higher }) \text { than baseline }+1}{\text { number of bootstrap resampling }(10000)+1}
$$




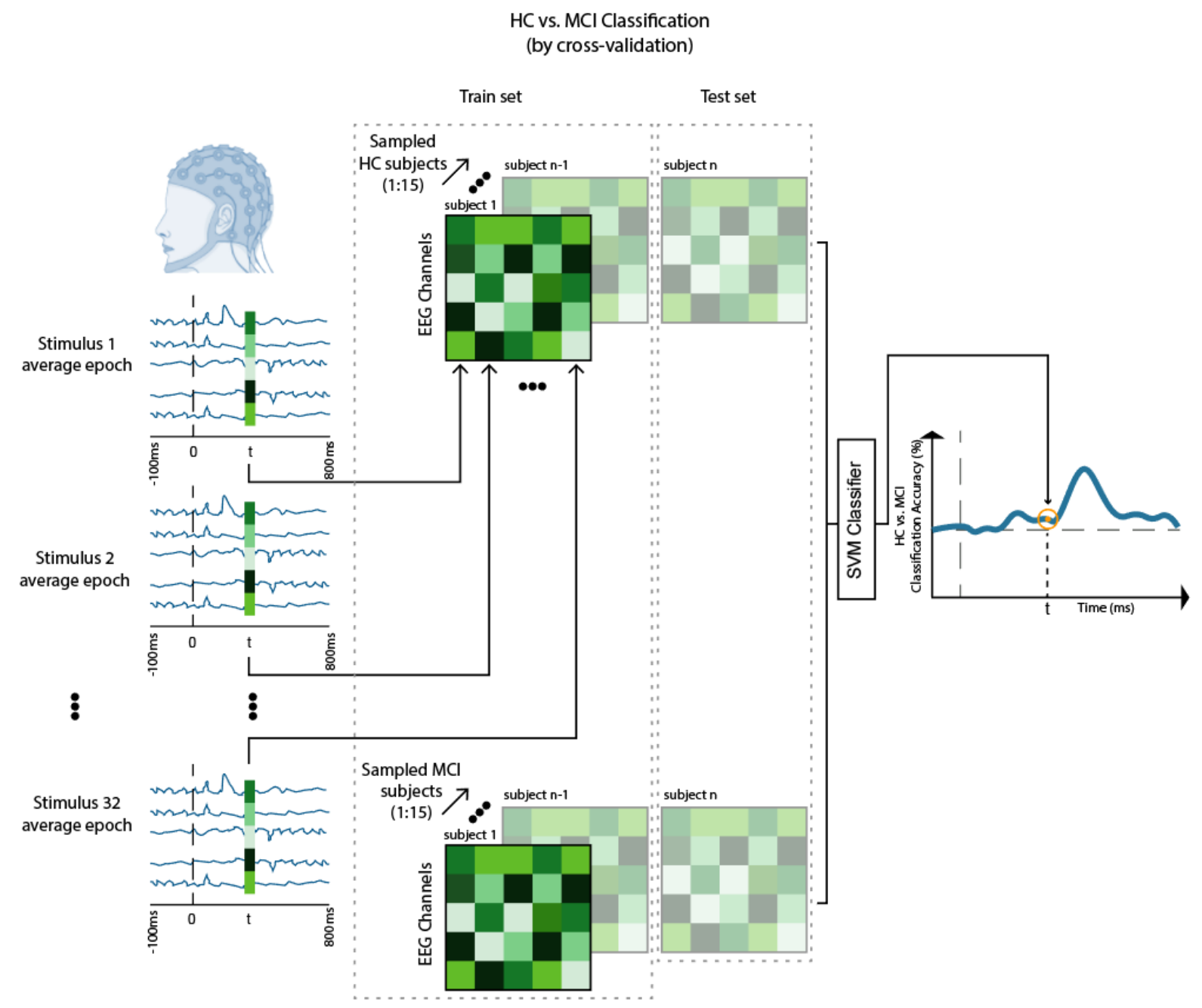

Figure 4. HC vs. MCl classification over time based on EEG response patterns. At each time-point, the pattern of EEG activation is a $63 \times 32$ matrix with columns being the EEG responses of channels to the 32 stimuli. We applied 10000 bootstrap resamplings (without replacement) of participants and each time trained a leave-one-out cross-validation SVM model to discriminate HC from MCl based on their EEG activation patterns.

\subsubsection{Permutation Test}

Permutation test consists of randomly relabeling the samples from two populations to form a null distribution. It computes a $p$-value by testing a target statistic against the null hypothesis:

$$
p-\text { value }=\frac{\text { number of members from the null distibution lower }(\text { or higher }) \text { than the target }+1}{\text { number of permutations }(10000)+1}
$$

\subsubsection{Rank-Sum}

Rank-sum (also known as Wilcoxon-Mann-Whitney test) tests the null hypothesis that the data in $x$ and $y$ are sampled from continuous distributions with equal medians, against the alternative that they are not (44). We used rank-sum to compute the $p$ - 
value when comparing $\mathrm{HC}$ and $\mathrm{MCl}$ medians of animal vs. non-animal decoding amplitude, ICA speed and accuracy and, mean of ERP responses.

\subsection{Data availability statement}

The data generated during this study are included in the article. Commercially insensitive raw data can be made available upon reasonable request from the corresponding author.

\section{Results}

\subsection{A reduction in the speed and accuracy of animacy processing in $\mathrm{MCl}$ patients}

We compared the neural speed and accuracy of animal/non-animal discrimination between healthy and $\mathrm{MCl}$. To this end, for each group, we computed the time at which animal images can best be discriminated from non-animals based on their EEG responses. This time-point is referred to as the peak of animal/non-animal decoding. $\mathrm{MCl}$ patients showed a median delay of $39 \mathrm{~ms}(95 \% \mathrm{Cl}=[8,111], \mathrm{SE}=34 \mathrm{~ms})$ in processing the animacy information in comparison to healthy individuals ( $p$-value $=0.0001 ; 10000$ bootstrap resampling of participants, Figure 5a). Additionally, in this decoding peak timepoint, the neural accuracy of animal detection was significantly lower in $\mathrm{MCl}$ patients compared to healthy controls $(\mathrm{HC})$ ( $p$-value $=0.018$; rank-sum, Figure $5 b)$.

The representational dissimilarity method is a way to represent patterns of brain information processing (45). To study the neural representation underlying animacy categorization, we compared the representational dissimilarity matrices (RDM) and ERP responses (Figures 5c, 5d) of the two groups at the time of each individual's animal vs. non-animal decoding peak.

The $\mathrm{HC} / \mathrm{MCl} \mathrm{RDM}$ in the peak animacy decoding time-point represents the pattern of EEG responses at a time-point in which the brain representation of animal images is best separated from that of non-animals (Figure $5 \mathrm{c}$ ). While the peak animacy time-point was delayed for the $\mathrm{MCl}$ group, the $\mathrm{MCl}$ RDM was not significantly different from that of the $\mathrm{HC}$ RDM in its peak, suggesting that it took more time for the $\mathrm{MCl}$ patients to converge to a pattern similar to that of the $\mathrm{HC}$, which is used for discriminating animals from nonanimals. 
bioRxiv preprint doi: https://doi.org/10.1101/2020.11.20.390435; this version posted February 7, 2021. The copyright holder for this preprint (which was not certified by peer review) is the author/funder, who has granted bioRxiv a license to display the preprint in perpetuity. It is made available under aCC-BY-NC 4.0 International license.

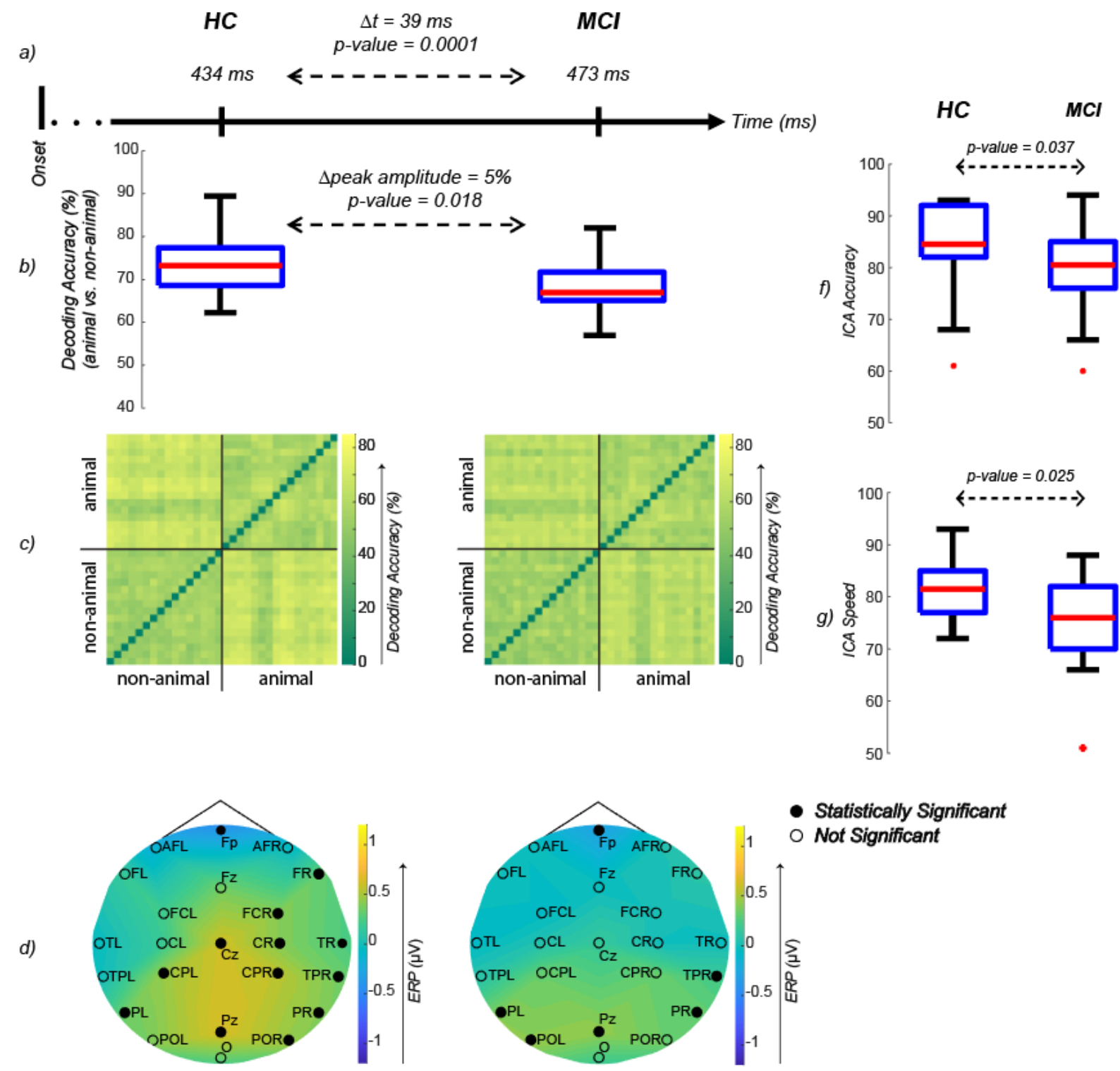

Figure 5. a) Median of animal vs. non-animal decoding accuracy peak time-points. 10000 bootstrap resampling without replacement of participants was applied to measure the differences between $\mathrm{HC}$ and $\mathrm{MCl}$. The median of time differences between $\mathrm{MCl}$ and $\mathrm{HC}$ is 39ms ( $p$-value = 0.0001; One-sided bootstrap test) b) Box-plot of animal vs. nonanimal EEG decoding peak amplitude. The difference of $\mathrm{HC}$ and $\mathrm{MCl}$ amplitude medians is 5\% ( $p$-value=0.018; ranksum). c) Mean EEG RDM of participants at the time of animal vs. non-animal decoding peak. No significant difference was observed between the RDMs (permutation test). d) Mean ERP of participants at the time of animal vs. non-animal decoding peak. The black dots indicate significant activation in channels of the specified region (FDR-corrected at 0.05 sign-rank). No significant difference between the ERP of HC and MCI was observed (FDR-Corrected at 0.05 rank-sum). f) Box-plot of the ICA test accuracy (results of the behavioral ICA, taken on iPad). A significant difference is observed between $\mathrm{HC}$ and $\mathrm{MCl}$ (p-value = 0.037; rank-sum). e) Box-plot of the ICA test speed (results of the behavioral ICA, taken on iPad). A significant difference is observed between $\mathrm{HC}$ and $\mathrm{MCl}$ ( $p$-value $=0.025$; rank-sum).

Fp: prefrontal; AFL: left anterior frontal; AFR: right anterior frontal; FL left frontal; Fz: midline frontal; FR: right frontal; FCL: left fronto-central; FCR: right fronto-central; TL: left temporal; CL: left central; Cz: midline central; CR: right central; TR: right temporal; TPL: left temporo-parietal; CPL: left centro-parietal; CPR: right centro-parietal; TPR: right temporoparietal; PL: left parietal; PR: right parietal; POL: left parieto-occipital; Pz: midline parietal; POR: right parieto-occipital; O: occipital

We also looked at the ERP responses at these peak time-points between $\mathrm{HC}$ and $\mathrm{MCl}$ : 


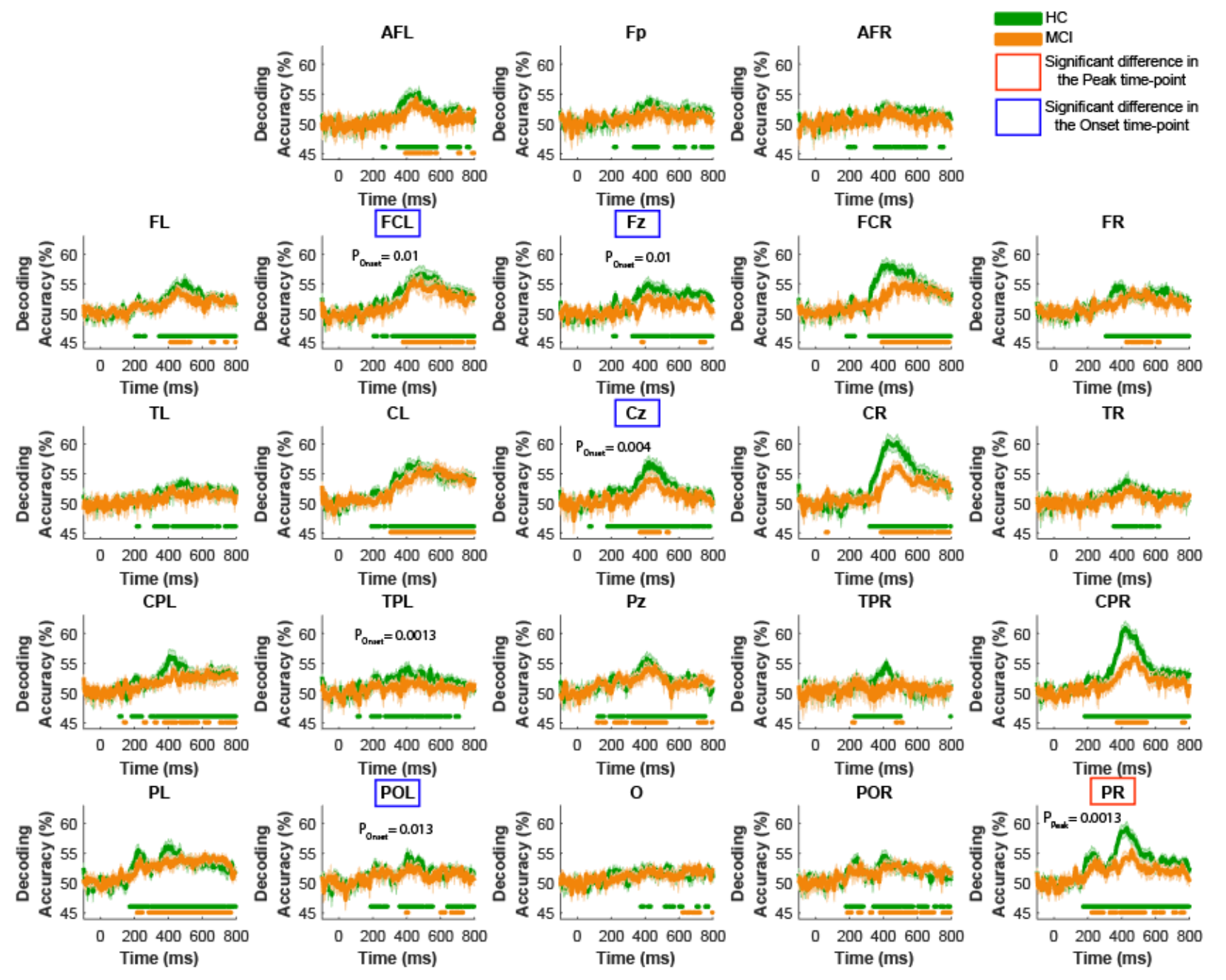

Figure 6. Animal vs. non-animal decoding time course across groups of EEG channels. Horizontal color dots indicate time-points with significant decoding accuracy in the corresponding group (green for $\mathrm{HC}$ and orange for $\mathrm{MCl}$ ). In regions specified with red rectangles MCl's peak animacy decoding time-point is significantly later than that of HC; in regions specified with blue rectangles MCl's onset of animacy decoding significance is significantly later than that of HC (FDRcorrected at 0.05 ; bootstrap test with 10,000 resampling of participants on $\mathrm{MCl}$ minus $\mathrm{HC}$ time-points).

channels in the right and left parietals, right temporal-parietal, midline parietal, and midline frontal lobes were significantly activated in both groups. HC showed significant activation levels in the right parieto-occipital, right temporal, and central lobes (midline central, right and left central-parietals, right central, and fronto-central); however, these were absent from the $\mathrm{MCl}$ brain activation map. On the other hand, only $\mathrm{MCl}$ patients showed significant activation on the left parieto-occipital lobe.

Analyzing the behavioral data of participants while taking the ICA test (on an iPad outside the EEG), we found the results to be consistent with the findings from the EEG data: the speed and accuracy of animacy detection, as measured by the ICA test, were also significantly deteriorated in patients with $\mathrm{MCl}$ ( $p$-value $=0.025$ and $p$-value $=0.037$ respectively) (Figures 5f, 5g). 
a)
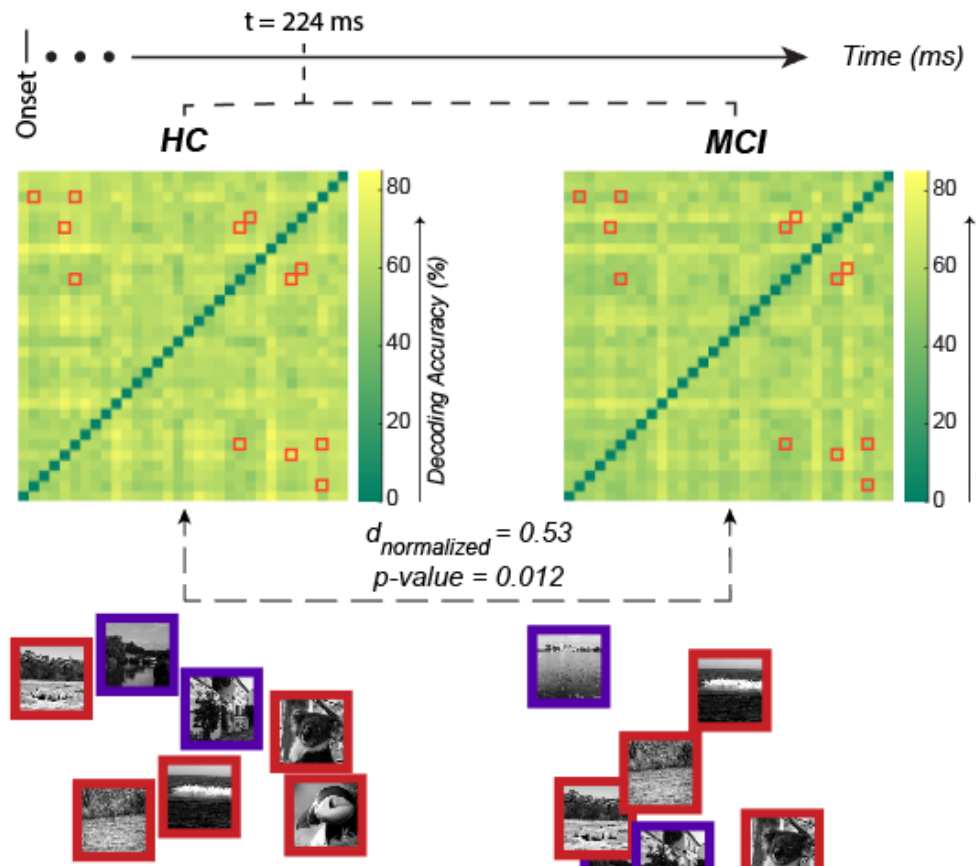

antoris

d)

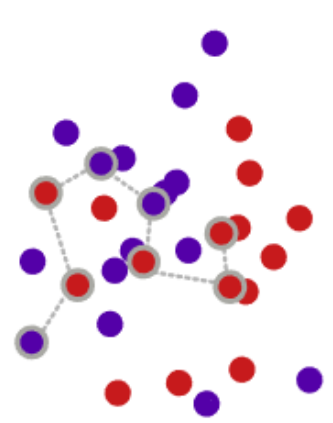

$\mathrm{MCl}$
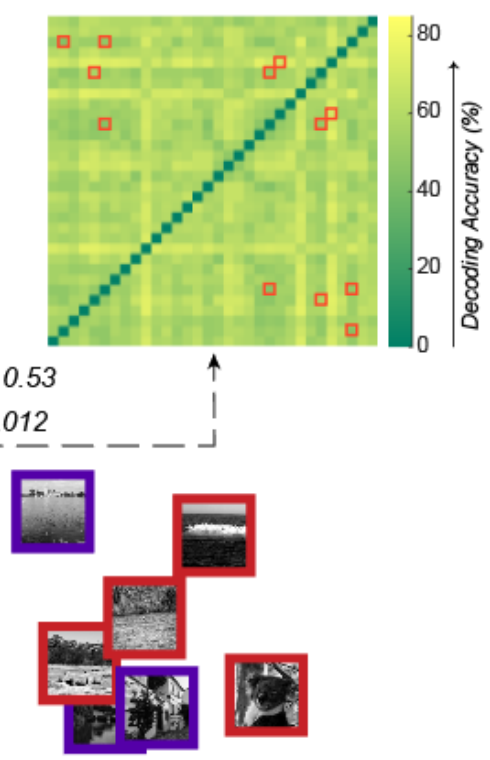

animal

non-animal

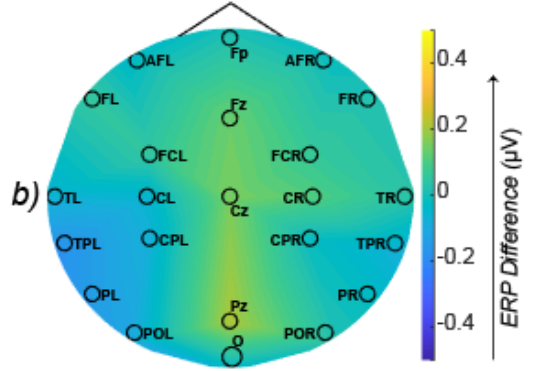

HC ERP minus $\mathrm{MCl}$ ERP (no significant difference)

Figure 7. a) The RDM of $\mathrm{HC}$ and $\mathrm{MCl}$ at the time-point of maximum Euclidean distance ( $t=224 \mathrm{~ms}, d=146.6, p$-value $=0.012$, permutation test). We further normalized the Euclidean distance to make it more meaningful by fitting a logarithmic function on the maximum distance (222.7), baseline distance (115.7) and the minimum distance (0). The logarithmic function scales the distances to the [0, 1] interval, and the observed distance between RDMs becomes 0.53. The highlighted elements (i.e. red-squares) of the RDMs are the pairwise dissimilarities with a significant difference between $\mathrm{HC}$ and $\mathrm{MCl}$ (FDR-corrected at 0.05 rank-sum). b) Difference of the mean ERPs across EEG channels (HC minus MCl) at $t=224 m s$. None of the EEG channels show a significant difference (all $p$-values $>0.05$ ) between $\mathrm{HC}$ and $\mathrm{MCl}$ at this time-point. c) MDS of the stimuli that showed a significant difference in their pairwise dissimilarity between $\mathrm{HC}$ and $\mathrm{MCl}$ (those specified by red squares in the two RDMs). d) MDS of all the stimuli. The dots that are connected with dashed lines are the same stimuli shown in panel 'c'.

We also examined the channel-specific animacy decoding time courses to investigate whether there is a significant delay in the peak and/or the onset of animacy processing in $\mathrm{MCl}$ patients at the level of EEG channels (Figure 6). We found that there is a significant delay in the animacy decoding peak time-point of $\mathrm{MCl}$ patients in the right parietal lobe compared to $\mathrm{HC}$ ( $p$-value $=0.0013$, Figure 6 ). Additionally, the significance of animal vs. 
a) $\mathrm{HC}$ and MCI ERP Difference

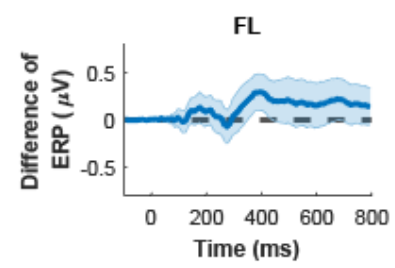

PL
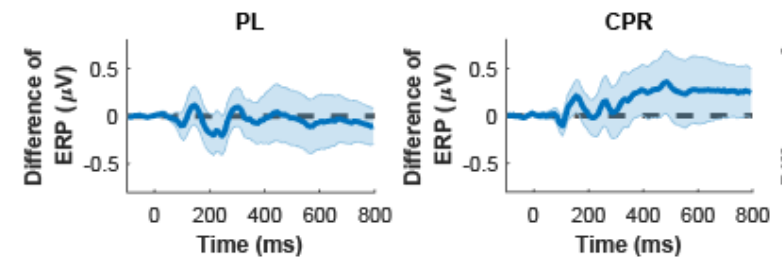

b) $\mathrm{HC}$ vs. MCl Pattern Classification

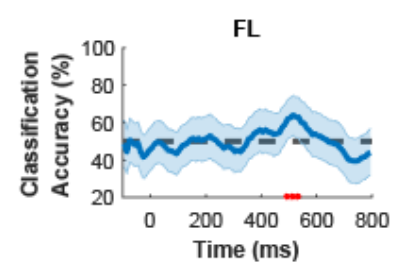

PL
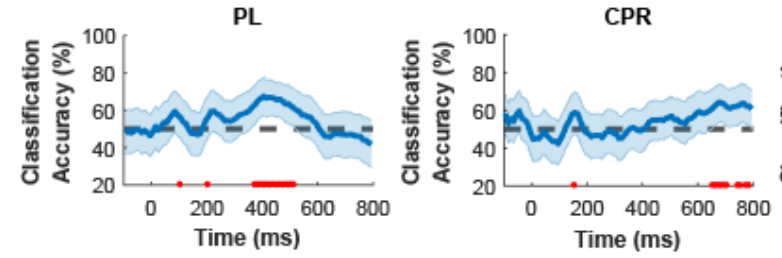

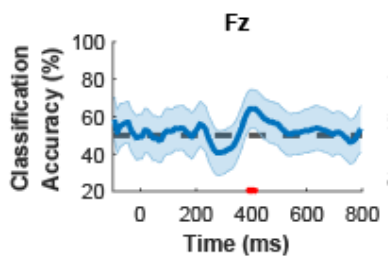

CPR
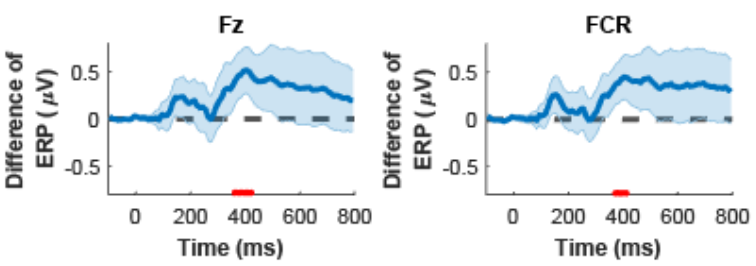

TR

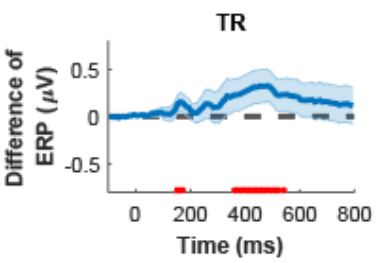

CR

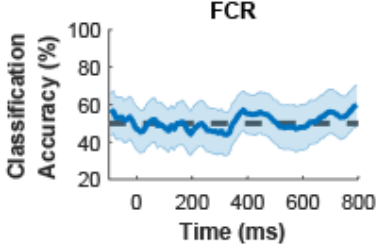

TR

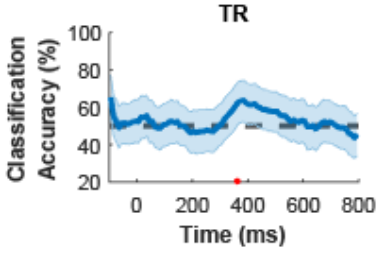

Figure 8. a) $\mathrm{HC}$ and. $\mathrm{MCl}$ ERP differences in regions where either the ERP difference or the HC vs. $\mathrm{MCl}$ classification were statistically significant. The shaded error bars indicate $95 \%$ confidence interval of the ERP difference ( $\mathrm{HC}$ minus $\mathrm{MCl}$ ). Red dots indicate time-points with significant level of difference in ERP (FDR-corrected at 0.05 across time; rank-sum). b) The $\mathrm{HC}$ vs. $\mathrm{MCl}$ classification based on the pattern of EEG data (i.e. channels $x$ stimuli), in regions where either the ERP difference or the $H C$ vs. $\mathrm{MCl}$ classification of EEG responses were statistically significant. The shaded error bars indicate 95\% confidence intervals. Red dots indicate time-points with significant $\mathrm{HC}$ vs. $\mathrm{MCl}$ classification accuracy (FDRcorrected at 0.05; 10000 bootstrap resampling of participants).

$F L$ : left frontal; Fz: midline frontal; FCR: right fronto-central; PL: left parietal; CPR: right central parietal; TR: right temporal;

non-animal decoding started later in the $\mathrm{MCl}$ patients in EEG channels of left frontocentral, midline frontal, midline central, and left parieto-occipital lobes (Figure 6).

\subsection{Comparing Patterns of Visual Information Processing in $\mathrm{HC}$ and $\mathrm{MCl}$}

We compared patterns of visual information processing in $\mathrm{HC}$ and $\mathrm{MCl}$ using their RDMs over time. The maximum difference between $\mathrm{HC}$ and $\mathrm{MCI}$ RDMs was observed at $\mathrm{t}=$ $224 \mathrm{~ms}$ after the stimulus onset (scaled Euclidean distance, $\mathrm{d}=0.53$ [out of 1], $\mathrm{p}$-value $=$ 0.012; Figure 7a). At this time-point ( $\mathrm{t}=224 \mathrm{~ms}$ ), an SVM classifier (leave-one-out crossvalidation) could significantly discriminate between $\mathrm{HC}$ and $\mathrm{MCl}$ patterns of visual information (represented by their RDMs) with an accuracy of $70.4 \%$ ( $p$-value $=0.0036$, 10,000 bootstrap sampling of participants).

We also looked at the univariate differences between $\mathrm{HC}$ and $\mathrm{MCl}$ at the same time-point (i.e., $\mathrm{t}=224 \mathrm{~ms}$ ); there was no significant difference between the two groups in their ERP responses (Figure $7 b$ ). This demonstrates that the response patterns carry valuable information above and beyond what is captured in the neural activity's mean responses. 
Furthermore, examining the $\mathrm{HC}$ and $\mathrm{MCI}$ RDMs, we highlighted RDM cells that were significantly different between the two groups (Figure 7a). Each RDM cell represents the difference between the EEG patterns of the two stimuli at a given time-point. To provide a more intuitive understating of the differences in patterns, we used multidimensional scaling (MDS) to visualize the RDMs on a 2D surface. Figure 7c illustrates the stimuli with a significant difference between $\mathrm{HC}$ and $\mathrm{MCl}$, and Figure $7 \mathrm{~d}$ visualizes all the stimuli.

\subsection{Temporal Dynamics of Animacy Categorization}

To study the temporal dynamics of animacy categorization in $\mathrm{HC}$ and $\mathrm{MCl}$, we compared the mean response (i.e., ERP) as well as EEG activation patterns (i.e., 63 channels $\times 32$ stimuli matrices) between $\mathrm{HC}$ and $\mathrm{MCl}$ over time. For each $\mathrm{EEG}$ channel group, we measured the ERP differences between $\mathrm{HC}$ and $\mathrm{MCl}$ over time: midline frontal, right fronto-central, and right temporal regions showed a significant difference (Figure 8a). We also performed a classification over the EEG activation patterns to see if $\mathrm{HC}$ and $\mathrm{MCl}$ can be discriminated based on their epoched EEG responses. $\mathrm{HC}$ and $\mathrm{MCl}$ could be discriminated based on their EEG activation patterns in the left frontal, midline frontal, left parietal, and right central parietal lobes (Figure 8b).

Looking at the EEG data (Figure 8), we found that $\mathrm{HC}$ and $\mathrm{MCl}$ could be discriminated starting from $375 \mathrm{~ms}$ in the left parietal (PL) and from $495 \mathrm{~ms}$ in the left frontal (FL) both to $515 \mathrm{~ms}$ after the stimulus onset only based on their patterns of activity, but not the ERPs. Additionally, the pattern of activity in centro-parietal (CPR) could discriminate $\mathrm{HC}$ from $\mathrm{MCl}$ in almost every time-points after $\mathrm{t}=655 \mathrm{~ms}$. On the other hand, the ERP responses showed a significant difference between $\mathrm{HC}$ and $\mathrm{MCl}$ in the right fronto-central (FCR) at around $\mathrm{t}=405 \mathrm{~ms}$ and in the right temporal (TR) from $155 \mathrm{~ms}$ to $174 \mathrm{~ms}$ and $365 \mathrm{~ms}$ to $545 \mathrm{~ms}$. At the same time-points the two groups could not be separated based on their activation patterns. The midline frontal $(\mathrm{Fz})$ was the only region that could differentiate $\mathrm{HC}$ and $\mathrm{MCl}$ based on both the ERP responses and the activation patterns at around $\mathrm{t}=405 \mathrm{~ms}$ after the stimulus onset.

In the previous subsection, we demonstrated that at $\mathrm{t}=224 \mathrm{~ms}$, the difference between $\mathrm{HC}$ and $\mathrm{MCl}$ in the EEG response patterns (captured by RDM) was at its maximum, while the level of activity (captured by ERP) remains unchanged. Here, we identified five groups of channels whose EEG data could discriminate between $\mathrm{HC}$ and $\mathrm{MCl}$, either based on the activation patterns or the ERP responses, but not both. This is consistent with the reported results in the previous section (3.2), demonstrating that EEG activation patterns could be different even though there might be no difference at the level of ERP. 


\section{Discussion}

In this study, we investigated the temporal dynamics of animacy visual processing in patients with $\mathrm{MCl}$ and argued that the speed of processing animacy information is a potential biomarker for detecting $\mathrm{MCl}$. The proposed rapid visual categorization task in the ICA test is more challenging than the typical memory tasks, making it more sensitive to less severe brain deteriorations.

Previous resting-state and task-based EEG studies have focused on univariate changes (e.g., ERP, frequency bands, connectivity) in patients with $\mathrm{MCl}$ and individuals with mild to moderate $A D(5-7,12-14,46)$. Here, using a rapid visual categorization task and applying multivariate pattern analysis, we looked beyond the univariate changes and studied the categorical representation of animacy information in the brain of old healthy individuals and patients with $\mathrm{MCl}$. We demonstrated that patients with $\mathrm{MCl}$ could be discriminated from $\mathrm{HC}$ based on their pattern of animacy representation. Furthermore, we identified regions in which either the mean EEG responses or the pattern of brain activity show significant differences between $\mathrm{HC}$ and $\mathrm{MCl}$.

One of the study limitations is the relatively small number of participants in comparison to large clinical studies. Future work can investigate how well the current results generalize to cohorts of larger MCl/AD patients.

\subsection{Task Differences in EEG studies of $\mathrm{HC}$ and $\mathrm{MCl}$}

Consistent with previous reports in resting-state EEG studies (47) and studies with a visual memory task (14), we observed univariate differences between $\mathrm{HC}$ and $\mathrm{MCl}$ in the temporal and the fronto-central electrodes.

In contrast, we did not find any significant difference in ERP responses of $\mathrm{HC}$ vs. $\mathrm{MCl}$ in centro-parietal and parietal electrodes -which is reported previously in an EEG study with a visual working memory task (13). We also observed no univariate difference in the frontal and occipital electrodes, while previous resting-state EEG studies have reported differences between $\mathrm{HC}$ and $\mathrm{MCI}$ in these regions $(47,48)$. On the other hand, we found that $\mathrm{MCl}$ patients could be discriminated from $\mathrm{HC}$ based on their ERP responses of the midline frontal region electrodes. These differences could potentially be explained by the difference in the tasks used for each of these studies (i.e., visual working memory and resting-state vs. rapid visual categorization).

In addition to the previous univariate findings, here we revealed multivariate differences between $\mathrm{HC}$ and $\mathrm{MCl}$ in their patterns of EEG responses: midline frontal, left frontal, left 
parietal and right centro-parietal electrodes showed significant multivariate differences between $\mathrm{HC}$ and $\mathrm{MCl}$. Furthermore, the categorical representation of animacy information of the right parietal electrode emerged later in the $\mathrm{MCl}$ patients compared to that of $\mathrm{HC}$. Also, in comparison with $\mathrm{HC}$, the $\mathrm{MCl}$ patients had significantly longer onset latencies of animacy information in the left fronto-central, midline frontal, midline central, and left parieto-occipital electrodes.

\subsection{Neural Speed of Information Processing in $\mathrm{MCl}$ patients}

Rapid recognition of animate objects is a fundamental ability of human visual cognition. Previous M/EEG studies have investigated the temporal neural dynamics of animacy processing in young healthy individuals. Using slightly different visual tasks and stimuli, studies have shown that the onset and peak of animacy decoding emerges between $66 \mathrm{~ms}$ to $157 \mathrm{~ms}$ (32); or from $80 \mathrm{~ms}$ to $240 \mathrm{~ms}$ (33) after the stimulus onset. Here, we showed that in old healthy individuals, the onset and the peak of animacy decoding emerges between $131 \mathrm{~ms}(\mathrm{SE}=30)$ and $434 \mathrm{~ms}(\mathrm{SE}=30)$ after the stimulus onset. This result indicates that normal aging causes a reduction in the animacy information processing speed (IPS). Compared to the old healthy individuals, animacy IPS was further delayed in $\mathrm{MCl}$ patients (onset of animacy decoding: 196ms \pm 16 , peak animacy decoding: $473 \mathrm{~ms} \pm 34$ after the stimulus onset). These findings confirm a significant decrease in the speed of neural information processing in patients with $\mathrm{MCl}$ and are consistent with previous behavioral studies showing a decline in visual IPS in $\mathrm{MCl}$ patients compared to $\mathrm{HC}(35,49)$. Together, these results suggest the IPS as a potential biomarker for the early detection of $A D$, along with memory symptoms or ideally before the onset of memory symptoms.

\section{Acknowledgement}

We thank the National Brain Mapping Laboratory (NBML), where all the EEG data acquisitions were done. Authors are also grateful to Mohammad Mohaghar for proof reading the manuscript. 


\section{References}

1. Petersen RC, Caracciolo B, Brayne C, Gauthier S, Jelic V, Fratiglioni L. Mild cognitive impairment: a concept in evolution. J Intern Med. 2014 Mar;275(3):21428.

2. 2018 Alzheimer's disease facts and figures. Alzheimer's \& Dementia: The Journal of the Alzheimer's Association. 2018 Mar 1;14(3):367-429.

3. Ward A, Tardiff S, Dye C, Arrighi HM. Rate of conversion from prodromal Alzheimer's disease to Alzheimer's dementia: a systematic review of the literature. Dement Geriatr Cogn Dis Extra. 2013;3(1):320-32.

4. Dunne RA, Aarsland D, O'Brien JT, Ballard C, Banerjee S, Fox NC, et al. Mild cognitive impairment: the Manchester consensus. Age Ageing [Internet]. $2020 \mathrm{Nov}$ [cited 2020 Nov 29]; Available from: https://academic.oup.com/ageing/advancearticle/doi/10.1093/ageing/afaa228/5960421

5. Vecchio F, Miraglia F, Marra C, Quaranta D, Vita MG, Bramanti P, et al. Human brain networks in cognitive decline: a graph theoretical analysis of cortical connectivity from EEG data. J Alzheimers Dis. 2014;41(1):113-27.

6. Babiloni C, Lizio R, Marzano N, Capotosto P, Soricelli A, Triggiani Al, et al. Brain neural synchronization and functional coupling in Alzheimer's disease as revealed by resting state EEG rhythms. International Journal of Psychophysiology. 2016 May 1;103:88-102.

7. Pijnenburg $Y$ a. L, v d Made $Y$, van Cappellen van Walsum AM, Knol DL, Scheltens $P$, Stam CJ. EEG synchronization likelihood in mild cognitive impairment and Alzheimer's disease during a working memory task. Clin Neurophysiol. 2004 Jun;115(6):1332-9.

8. Moretti DV, Frisoni GB, Binetti G, Zanetti O. Anatomical Substrate and Scalp EEG Markers are Correlated in Subjects with Cognitive Impairment and Alzheimer's Disease. Front Psychiatry [Internet]. 2011 [cited 2020 Sep 28];1. Available from: https://www.frontiersin.org/articles/10.3389/fpsyt.2010.00152/full

9. Poil S-S, De Haan W, van der Flier WM, Mansvelder HD, Scheltens P, LinkenkaerHansen K. Integrative EEG biomarkers predict progression to Alzheimer's disease 
at the $\mathrm{MCl}$ stage. Front Aging Neurosci [Internet]. 2013 [cited 2020 Sep 28];5. Available from: https://www.frontiersin.org/articles/10.3389/fnagi.2013.00058/full

10. Babiloni C, Del Percio C, Lizio R, Noce G, Cordone S, Lopez S, et al. Abnormalities of cortical neural synchronization mechanisms in patients with dementia due to Alzheimer's and Lewy body diseases: an EEG study. Neurobiology of Aging. 2017 Jul 1;55:143-58.

11. Vecchio F, Miraglia F, Piludu F, Granata G, Romanello R, Caulo M, et al. "Small World" architecture in brain connectivity and hippocampal volume in Alzheimer's disease: a study via graph theory from EEG data. Brain Imaging and Behavior. 2017 Apr 1;11(2):473-85.

12. Missonnier P, Deiber M-P, Gold G, Herrmann FR, Millet P, Michon A, et al. Working memory load-related electroencephalographic parameters can differentiate progressive from stable mild cognitive impairment. Neuroscience. 2007 Dec $5 ; 150(2): 346-56$.

13. Fraga FJ, Mamani GQ, Johns E, Tavares G, Falk TH, Phillips NA. Early diagnosis of mild cognitive impairment and Alzheimer's with event-related potentials and event-related desynchronization in $\mathrm{N}$-back working memory tasks. Comput Methods Programs Biomed. 2018 Oct;164:1-13.

14. Fraga FJ, Ferreira LA, Falk TH, Johns E, Phillips ND. Event-related synchronisation responses to $\mathrm{N}$-back memory tasks discriminate between healthy ageing, mild cognitive impairment, and mild Alzheimer's disease. In: 2017 IEEE International Conference on Acoustics, Speech and Signal Processing (ICASSP). 2017. p. 964-8.

15. Han Y, Wang K, Jia J, Wu W. Changes of EEG Spectra and Functional Connectivity during an Object-Location Memory Task in Alzheimer's Disease. Front Behav Neurosci [Internet]. 2017 May 31 [cited 2020 Aug 12];11. Available from: https://www.ncbi.nlm.nih.gov/pmc/articles/PMC5449767/

16. Paik J-S, Ha M, Jung YH, Kim G-H, Han K-D, Kim H-S, et al. Low vision and the risk of dementia: a nationwide population-based cohort study. Scientific Reports. 2020 Jun 4;10(1):9109.

17. Cogan DG. Visuospatial dysgnosia. Am J Ophthalmol. 1979 Sep;88(3 Pt 1):3618.

18. Jackson GR, Owsley C. Visual dysfunction, neurodegenerative diseases, and aging. Neurologic Clinics. 2003 Aug 1;21(3):709-28. 
19. Tzekov R, Mullan M. Vision function abnormalities in Alzheimer disease. Surv Ophthalmol. 2014 Aug;59(4):414-33.

20. Sadun AA, Borchert M, DeVita E, Hinton DR, Bassi CJ. Assessment of Visual Impairment in Patients With Alzheimer's Disease. American Journal of Ophthalmology. 1987 Aug 1;104(2):113-20.

21. Cogan DG. Visual Disturbances With Focal Progressive Dementing Disease. American Journal of Ophthalmology. 1985 Jul 1;100(1):68-72.

22. van Deursen JA, Vuurman EFPM, Verhey FRJ, van Kranen-Mastenbroek VHJM, Riedel WJ. Increased EEG gamma band activity in Alzheimer's disease and mild cognitive impairment. J Neural Transm. 2008 Sep;115(9):1301-11.

23. Briels CT, Eertink JJ, Stam CJ, van der Flier WM, Scheltens P, Gouw AA. Profound regional spectral, connectivity and network changes reflect visual deficits in Posterior Cortical Atrophy: an EEG study. Neurobiology of Aging [Internet]. 2020 Aug 5 [cited 2020 Sep 1]; Available from: http://www.sciencedirect.com/science/article/pii/S0197458020302463

24. Kiani R, Esteky H, Mirpour K, Tanaka K. Object Category Structure in Response Patterns of Neuronal Population in Monkey Inferior Temporal Cortex. Journal of Neurophysiology. 2007 Jun 1;97(6):4296-309.

25. Kriegeskorte N, Mur M, Ruff DA, Kiani R, Bodurka J, Esteky H, et al. Matching Categorical Object Representations in Inferior Temporal Cortex of Man and Monkey. Neuron. 2008 Dec 26;60(6):1126-41.

26. Connolly AC, Guntupalli JS, Gors J, Hanke M, Halchenko YO, Wu Y-C, et al. J Neurosci. 2012 Feb 22;32(8):2608-18.

27. Khaligh-Razavi S-M, Kriegeskorte N. Deep Supervised, but Not Unsupervised, Models May Explain IT Cortical Representation. PLOS Computational Biology. 2014 Nov 6;10(11):e1003915.

28. Khaligh-Razavi S-M, Henriksson L, Kay K, Kriegeskorte N. Fixed versus mixed RSA: Explaining visual representations by fixed and mixed feature sets from shallow and deep computational models. Journal of Mathematical Psychology. 2017 Feb 1;76:184-97.

29. Naselaris T, Stansbury DE, Gallant JL. Cortical representation of animate and inanimate objects in complex natural scenes. Journal of Physiology-Paris. 2012 Sep 1;106(5):239-49. 
30. Bacon-Macé N, Macé MJ-M, Fabre-Thorpe M, Thorpe SJ. The time course of visual processing: Backward masking and natural scene categorisation. Vision Research. 2005 May 1;45(11):1459-69.

31. Thorpe SJ. The Speed of Categorization in the Human Visual System. Neuron. 2009 Apr 30;62(2):168-70.

32. Cichy RM, Pantazis D, Oliva A. Resolving human object recognition in space and time. Nat Neurosci. 2014 Mar;17(3):455-62.

33. Carlson T, Tovar DA, Alink A, Kriegeskorte N. Representational dynamics of object vision: The first 1000 ms. Journal of Vision. 2013 Aug 1;13(10):1-1.

34. Khaligh-Razavi S-M, Cichy RM, Pantazis D, Oliva A. Tracking the Spatiotemporal Neural Dynamics of Real-world Object Size and Animacy in the Human Brain. Journal of Cognitive Neuroscience. 2018;30:1559-76.

35. Khaligh-Razavi S-M, Habibi S, Sadeghi M, Marefat H, Khanbagi M, Nabavi SM, et al. Integrated Cognitive Assessment: Speed and Accuracy of Visual Processing as a Reliable Proxy to Cognitive Performance. Sci Rep [Internet]. 2019 Jan 31 [cited 2019 Jul 25];9. Available from: https://www.ncbi.nlm.nih.gov/pmc/articles/PMC6355897/

36. Kalafatis C, Khaligh Razavi S-M. F3-01-03: the integrated cognitive assessment employing artificial intelligence for the detection of cognitive impairment. Alzheimer's \& Dementia. 2019 Jul 1;15(7S_Part_16):P864-P864.

37. Kalafatis $\mathrm{C}$, Modarres MH, Marefat $\mathrm{H}$, Khanbagi M, Karimi $\mathrm{H}$, Vahabi Z, et al. P4207: EMPLOYING ARTIFICIAL INTELLIGENCE IN THE DEVELOPMENT OF A SELF- ADMINISTERED, COMPUTERISED COGNITIVE ASSESSMENT FOR THE ASSESSMENT OF NEURODEGENERATION. Alzheimer's \& Dementia. 2019 Jul 1;15:P1355-6.

38. Khaligh-Razavi SM, Habibi S. System for assessing mental health disorder. UK Intellect Prop Off. 2013;

39. Khaligh-Razavi S-M, Sadeghi M, Khanbagi M, Kalafatis C, Nabavi SM. A selfadministered, artificial intelligence (Al) platform for cognitive assessment in multiple sclerosis (MS). BMC Neurology. 2020 May 18;20(1):193.

40. Nasreddine ZS, Phillips NA, Bédirian V, Charbonneau S, Whitehead V, Collin I, et al. The Montreal Cognitive Assessment, MoCA: a brief screening tool for mild cognitive impairment. J Am Geriatr Soc. 2005 Apr;53(4):695-9. 
41. Mioshi E, Dawson K, Mitchell J, Arnold R, Hodges JR. The Addenbrooke's Cognitive Examination Revised (ACE-R): a brief cognitive test battery for dementia screening. Int J Geriatr Psychiatry. 2006 Nov;21(11):1078-85.

42. Tadel F, Baillet S, Mosher JC, Pantazis D, Leahy RM. Brainstorm: A User-Friendly Application for MEG/EEG Analysis [Internet]. Computational Intelligence and Neuroscience. 2011 [cited 2019 Jul 28]. Available from: https://www.hindawi.com/journals/cin/2011/879716/

43. Chang C-C, Lin C-J. LIBSVM: A library for support vector machines. ACM Trans Intell Syst Technol. 2011 May 6;2(3):27:1-27:27.

44. Nonparametric Statistical Inference. Boca Raton; 2010. 650 p.

45. Kriegeskorte N, Kievit RA. Representational geometry: integrating cognition, computation, and the brain. Trends Cogn Sci. 2013 Aug;17(8):401-12.

46. Missonnier P, Gold G, Fazio-Costa L, Michel J-P, Mulligan R, Michon A, et al. Early event-related potential changes during working memory activation predict rapid decline in mild cognitive impairment. J Gerontol A Biol Sci Med Sci. 2005 May;60(5):660-6.

47. Babiloni C, Binetti G, Cassetta E, Dal Forno G, Del Percio C, Ferreri F, et al. Sources of cortical rhythms change as a function of cognitive impairment in pathological aging: a multicenter study. Clinical Neurophysiology: Official Journal of the International Federation of Clinical Neurophysiology. 2006 Feb;117(2):25268.

48. Choi J, Ku B, You YG, Jo M, Kwon M, Choi Y, et al. Resting-state prefrontal EEG biomarkers in correlation with MMSE scores in elderly individuals. Scientific Reports. 2019 Jul 18;9(1):10468.

49. Ruiz-Rizzo AL, Bublak P, Redel P, Grimmer T, Müller HJ, Sorg C, et al. Simultaneous object perception deficits are related to reduced visual processing speed in amnestic mild cognitive impairment. Neurobiology of Aging. $2017 \mathrm{Jul}$ $1 ; 55: 132-42$. 